Chir Gastroenterol 2007;23:212

\section{Hauptthema Defäkationsstörungen}

Diagnostik der Entleerungsstörung Buchmann, P. (Zürich)

Klassifikation von Defäkationsstörungen Kreis, M.E. (München)

Laparoskopische Resektionsrektopexie - Indikation, Technik, Langzeitergebnisse Keller, R.; Fischer, F.; Kleemann, M.; Roblick, U.J.; Mirow, L.; Bruch, H.-P. (Lübeck)

Die STARR-Operation: Indikation, Technik und Ergebnisse Schwandner, O. (Regensburg)

Interdisziplinäres
Gespräch

Zertifizierte medizinische Forbildung

Original Articles

\section{Defäkationsstörungen}

Herold, A. (Gesprächsleiter) (Mannheim)

Postoperative Drainagen bei viszeralchirurgischen Elektiveingriffen - notwendig, erlaubt oder schädlich?

Schüle, S.; Lehnert, T. (Bremen)

The Effectiveness of Nitric Oxide Derivates in Hydatid Disease
Parsak, C.K.; Hanta, I.; Koltas, I.S.; Sakman, G.; Akcam, T.; Kuleci, S.; Alabaz, O. (Balcali)

The Role of Nasogastric Tube Insertion after Gastrectomy

Hsu, S.-D.; Yu, J.-C.; Chen, T.-W.; Chou, S.-J. (Taipei); Hsieh, H.-F. (Taoyuan); Chan, D.-C. (Taipei)

Neuroendocrine Differentiation in Primary Rectal Cancer: Immunhistology with Prognostic Impact?

Schwandner, O. (Regensburg); Hilbert, M.; Broll, R.; Bruch, H.P. (Lübeck)

Economical Strategies of Laparoscopic Splenectomy: A Chinese Single-Center Experience

Chen, B.; Wang, L.; Wang, K.; Zhang, G.; Zhang, H.; Hu, S. (Jinan)

Totally Implantable Access Ports: Approach via Femoral Vein Hsieh, H.-F.; Liu, C.-T. (Taoyuan); Chuang, C.-H.; Yu, J.-C. (Taipei); Wu, H.-S. (Changhua); Lin, C.-H. (Taipei)

\section{Case Reports}

Primary Malignant Melanoma of the Small Intestine: A Rare Case Report and Review of Literature

Liu, W.-H.; Cheng, M.-F.; Lee, H.-S.; Hsu, H.-M. (Taipei)

Ruptured Hepatic Artery Aneurysm: A Rare Fatal Cause of Obstructive Jaundice Yilmaz, C.; Erkan, A.; Erkan, N.; Kuvaki, B. (Izmir)

Ruptured Pancreaticoduodenal Artery Aneurysm Combination with Acute Calculus Cholecystitis

Wu, Y.-C.; Kung, W.-C.; Hsu, J.-W.; Chang, T.-H. (Kaohsiung)

Appendectomy Induced Hepatic Cerebrospinal Fluid Pseudocyst in a Shunted Patient Liu, W.-H.; Hsieh, C.-T.; Chiang, Y.-H.; Chen, G.-J. (Taipei) 